EPJ Web of Conferences 75, 02001 (2014)

DOI: $10.1051 /$ epjconf/ 20147502001

(c) Owned by the authors, published by EDP Sciences, 2014

\title{
Effect of the annealing temperature on dynamic and structural properties of $\mathrm{Co}_{2} \mathrm{FeAl}$ thin films
}

\author{
M. Belmeguenai ${ }^{1, \text { a }}$, H. Tuzcuoglu ${ }^{1}$, M.Gabor ${ }^{2}$, T. Petrisor jr ${ }^{2}$, C. Tiusan ${ }^{3}$, F. Zighem ${ }^{1}$, S. M. Chérif ${ }^{1}$, and P. Moch ${ }^{1}$ \\ ${ }^{1}$ LSPM (CNRS-UPR 3407), Université Paris 13, 99 avenue Jean-Baptiste Clément, 93430 Villetaneuse, France \\ ${ }^{2}$ Centre for Superconductivity, Spintronics and Surface Science, Technical University of Cluj-Napoca, Str. \\ Memorandumului No. 28 RO-400114 Cluj-Napoca, ROMANIA Sciences, \\ ${ }^{3}$ Institut Jean Lamour, CNRS, Université de Nancy, BP 70239, F- 54506 Vandœuvre, France
}

\begin{abstract}
Co}_{2} \mathrm{FeAl}$ (CFA) thin films have been deposited on thermally oxidized $\mathrm{Si}(001)$ substrates by magnetron sputtering using a Tantalum cap layer and were then ex-situ annealed at $415^{\circ} \mathrm{C}, 515^{\circ} \mathrm{C}$ and $615^{\circ} \mathrm{C}$ during 15 minutes in vacuum. X-rays diffraction indicates that films CFA are polycrystalline and exhibit an in-plane isotropy growth. Ferromagnetic resonance measurements, using a microstrip line (MS-FMR), reveal a huge interfacial perpendicular magnetic anisotropy and small in-plane uniaxial anisotropy both annealing temperature-dependent. The MS-FMR data also allow concluding that the gyromagnetic factor remains constant and that the exchange stiffness constant increases with annealing temperature. Finally, the FMR linewidth decreases with increasing annealing temperature due to the enhancement of the chemical order, and allow deriving a very low intrinsic damping parameter $\left(1.3 \times 10^{-3}\right.$ at $\left.615^{\circ} \mathrm{C}\right)$.
\end{abstract}

\section{Introduction}

Spintronics, which is an emerging technology, where electron spin is used as an information vector, is actually facing three big challenges. In fact, the critical current density for the current-induced magnetization switching [1], which is proportional to Gilbert damping constant $\alpha$ and to the inverse of the spin polarization, should be reduced to low values for realizing the high density magnetic random access memory. The injection and detection of spin-polarized current from ferromagnet to a semiconductor is another problem of spintronics due to the conductivity mismatch between the two materials $[2$, 3]. Achieving high magnetoresistance values is a third challenge for boosting the performance of spintronic devices. The ideal key technology of all these challenges is high spin polarized $(100 \%)$ materials with low damping parameters such as Co-base Heusler alloys.

$\mathrm{Co}_{2} \mathrm{FeAl}(\mathrm{CFA})$ is one of the Co-based Heusler alloys having a very high Curie temperature $(1000 \mathrm{~K})$ and is theoretically predicted to have a half-metallic character of their spin-split band structure. It can provide giant tunnelling magnetoresistance $(360 \%$ at room temperature) [4] when used as an electrode in magnetic tunnel junctions, which makes CFA promising for practical applications. However, their crystalline structure and their chemical order strongly influence their magnetic and structural properties. Therefore, an annealing process is required to initiate their crystallization and to induce their atomic ordering. Therefore, it is of great interest to investigate the annealing temperature $\left(\mathrm{T}_{\mathrm{a}}\right)$ effects on the structural and magnetic properties of CFA thin films.

The purpose of this paper is to use ferromagnetic resonance in microstrip line (MS-FMR) under an in-plane and out of plane applied magnetic field combined to vibrating sample magnetometer (VSM) to investigate the correlation between structural and magnetic properties of CFA thin films grown on $\mathrm{Si}$ substrate and annealed at different temperatures. A special attention will be given to the effect of $T_{a}$ on the FMR linewidth and damping constant.

\section{Sample and experimental set up}

CFA films were grown on $\mathrm{Si}(001) / \mathrm{SiO}_{2}$ substrates using a magnetron sputtering system with a base pressure lower than $3 \times 10^{-9}$ Torr. Prior to the deposition of the CFA films, a $4 \mathrm{~nm}$ thick $\mathrm{MgO}$ buffer layer was grown at room temperature (RT) by $\mathrm{rf}$ sputtering from a $\mathrm{MgO}$ polycrystalline target under an Argon pressure of 15 mTorr. Next, $10 \mathrm{~nm}$ and $50 \mathrm{~nm}$ thick CFA films were deposited at room temperature by dc sputtering under an Argon pressure of $1 \mathrm{mTorr}$, at a rate of $0.1 \mathrm{~nm} / \mathrm{s}$. Finally, the CFA films were capped with a $\mathrm{MgO}(4 \mathrm{~nm}) / \mathrm{Ta}(4 \mathrm{~nm})$ bilayer. After the growth of the stack, the structures were

\footnotetext{
a Corresponding author: belmeguenai.mohamed@univ-paris13.fr
} 
ex-situ annealed at different temperature $\left(415^{\circ} \mathrm{C}, 515^{\circ} \mathrm{C}\right.$ and $615{ }^{\circ} \mathrm{C}$ ) during 15 minutes in vacuum. As grown 50 $\mathrm{nm}$ thick films has also been prepared for comparison.

The structural properties of the samples have been characterized by X-ray diffraction (XRD) using a fourcircle diffractometer. Their magnetic static and dynamic properties have been studied by vibrating sample magnetometer (VSM) and microstrip ferromagnetic resonance (MS-FMR) [5], respectively.

\section{Structural properties}

In order to determine the crystal structure of the CFA films, we performed x-ray diffraction experiments in grazing incidence configuration (GIXRD). Figure 1 shows the diffraction pattern recorded for the $50 \mathrm{~nm}$ CFA thick film annealed at $615^{\circ} \mathrm{C}$.

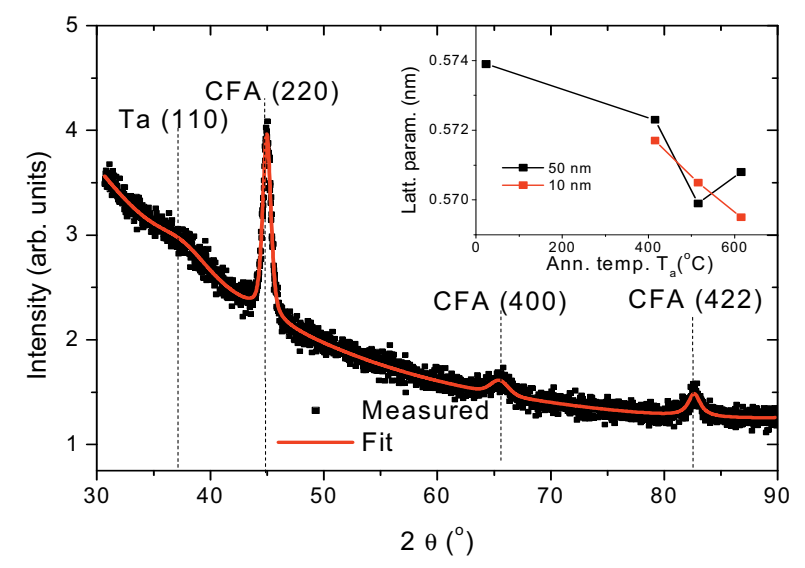

Fig. 1. (Color on line) X-ray diffraction pattern measured in grazing incidence geometry (GIXRD) for the $50 \mathrm{~nm}$ CFA thick film annealed at $615^{\circ} \mathrm{C}$. Symbols represent experimental data while lines are the result of the theoretical fit. The vertical dashed lines mark positions of the $\mathrm{Ta}(110)$ and $\mathrm{CFA}(220)$, (400), (422) reflections, respectively. The inset shows the evolution of lattice parameters, deduced from the position of the (220) peaks, with respect to the annealing temperature of $10 \mathrm{~nm}$ and $50 \mathrm{~nm} \mathrm{Co} 2 \mathrm{FeAl}$ films.

In spite of the relative low diffracted signal, the XRD pattern clearly shows peaks corresponding to the Ta (110) and CFA (220), (400) and (422) reflections, respectively. This indicates that the CFA film has a polycrystalline structure. The diffraction patterns exhibit peaks corresponding only to the $\mathrm{h}+\mathrm{k}+\mathrm{l}=4 \mathrm{n}$ type reflections which seems to indicate that the films show A2 structure with a disorder among $\mathrm{Co}, \mathrm{Fe}$, and $\mathrm{Al}$ sites. However, a precise evaluation of the chemical order is difficult to perform due to the relative low diffracted signal. The lattice parameter $(a)$, shown in inset of figure 1 , decreases with the increasing CFA thin films, due most probably to the enhancement of the chemical order, similar to the samples grown on $\mathrm{MgO}$ substrates where a direct relation between the annealing temperature and the chemical order has been observed [6]. Phi scan measurements (not shown here) indicate that the CFA films do not display any in-plane preferential growth direction.

\section{Magnetic characterizations}

All the measurements presented here have been made at room temperature and analysed using the model presented in [5].

\subsection{Static properties}

VSM hysteresis loops were measured versus external magnetic field orientations with respect to the substrate edge. Both the magnetization at saturation and the corresponding angular dependence of normalized remanent magnetizations $\left(\mathrm{M}_{\mathrm{r}} / \mathrm{M}_{\mathrm{s}}\right)$ have been extracted. The magnetization at saturation increases slightly with $\mathrm{T}_{\mathrm{a}}$ : for example, for $10 \mathrm{~nm}$ thick film, $\mathrm{M}_{\mathrm{s}}$ increases from $1000 \mathrm{emu} / \mathrm{cm}^{3}$ at $415^{\circ} \mathrm{C}$ to $1050 \mathrm{emu} / \mathrm{cm}^{3}$ for samples annealed at $615^{\circ} \mathrm{C}$.

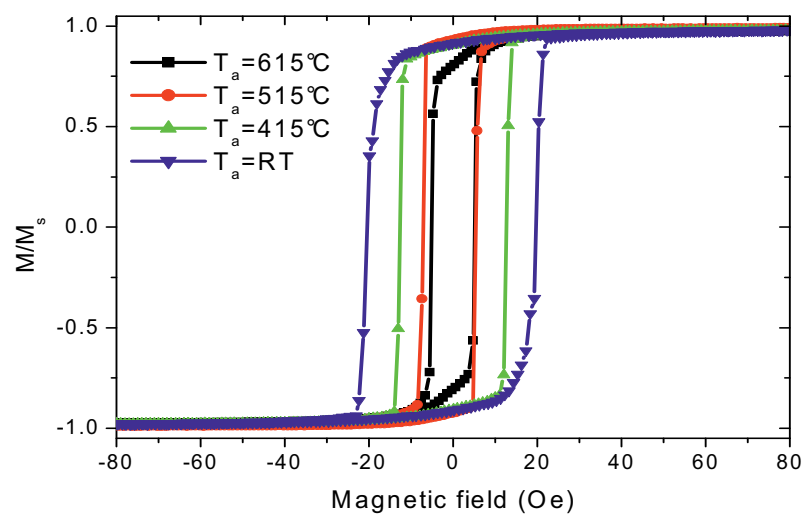

Fig. 2. (Color on line) Hysteresis loops, around the easy axis, of the $50 \mathrm{~nm}$ thick CFA films annealed at different temperatures.

Figure 2 shows typical hysteresis loops, around the easy axis, as function of the annealing temperature for the $50 \mathrm{~nm}$ thick CFA film. A clear different behaviour can be seen for the as grown sample and the film annealed at $415^{\circ} \mathrm{C}$. The increase of the coercive field $\left(\mathrm{H}_{\mathrm{c}}\right)$ with decreasing $T_{a}$ is another indication of the enhancement of the chemical order for the annealed samples. One should mention that the angular dependence of $\mathrm{M}_{\mathrm{r}} / \mathrm{M}_{\mathrm{s}}$ (not shown here) showed a uniaxial anisotropy behaviour for all the samples except the as grown one (annealed at RT) where a fourfold behaviour has been observed. Since the in-plane polycrystalline nature of samples, this fourfold behaviour is most probably due to a superposition of two perpendicular in-plane anisotropies due the inhomogeneities of the samples. As the annealing temperature increases the sample quality and the chemical order are enhanced and a unique uniaxial anisotropy is observed. This is confirmed by the variation of the uniaxial anisotropy easy axis direction with $\mathrm{T}_{\mathrm{a}}$. Moreover, magneto-optical Kerr effect hysteresis loops on different area of the samples revealed anisotropy dispersions due to inhomogeneities.

\subsection{Dynamic properties}

The uniform precession and the first perpendicular standing spin wave (PSSW) modes have been observed in perpendicular and in-plane applied field configurations 
for the $50 \mathrm{~nm}$ thick film while for the $10 \mathrm{~nm}$ thick film, the PSSW mode is not detected due to its high frequency over-passing the available bandwidth (0-24 GHz). Typical perpendicular field dependences of the resonance frequencies of the uniform and the PSSW modes are shown on figure 3 . By fitting the data in figure 3 to the model presented in [5], the gyromagnetic factor $(\gamma)$, the exchange stiffness constant $\left(\mathrm{A}_{\mathrm{ex}}\right)$ and the effective magnetization $\left(4 \pi \mathrm{M}_{\mathrm{eff}}\right)$ are extracted. The fitted $\gamma / 2 \pi=29.2 \mathrm{GHz} / \mathrm{T}$ is independent on $\mathrm{T}_{\mathrm{a}}$ while $\mathrm{A}_{\mathrm{ex}}$ decreases with $T_{a}$ suggesting an enhancement of the chemical order if the annealing temperature increases.

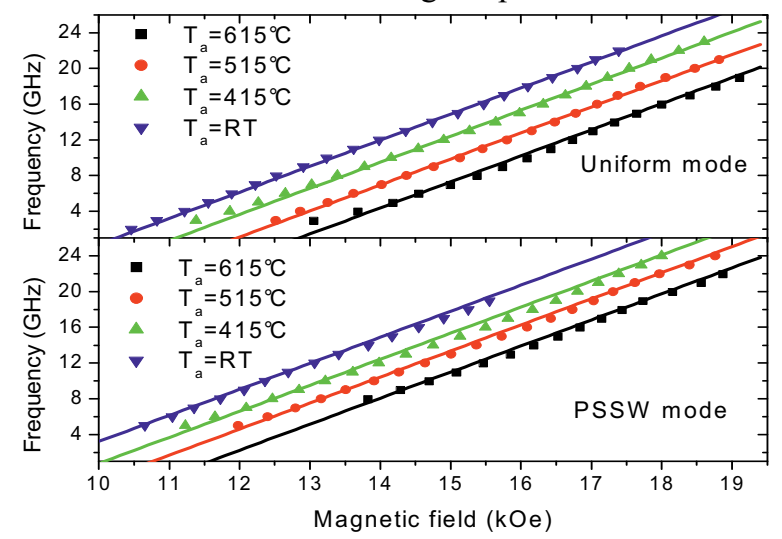

Fig. 3. (Color on line) Variation of the frequencies of the uniform and the PSSW modes for $50 \mathrm{~nm}$ thick CFA film annealed at different temperatures. Solid lines indicate the fit using the model described in [5].

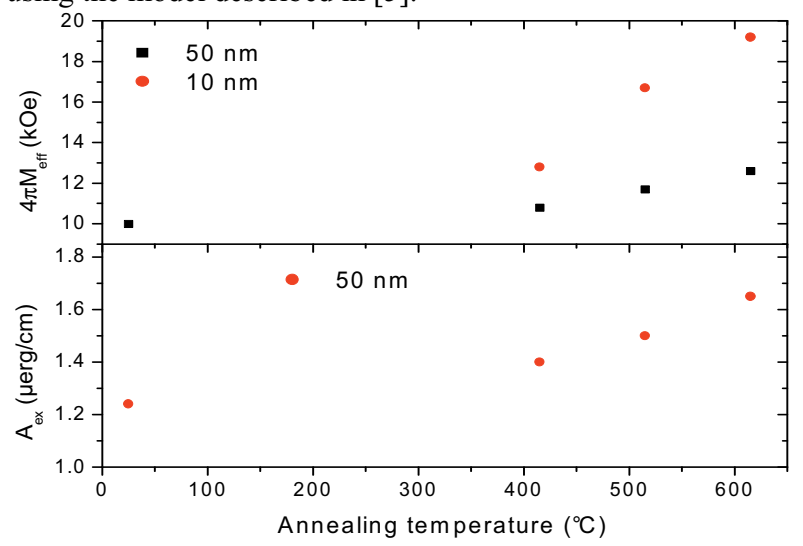

Fig. 4. (Color on line) Variations of the effective magnetization $\left(4 \pi \mathrm{M}_{\mathrm{eff}}\right)$ and the exchange stiffness constant $\left(\mathrm{A}_{\mathrm{ex}}\right)$, as function of the annealing temperature, of $10 \mathrm{~nm}$ and $50 \mathrm{~nm}$ thick CFA films.

The effective magnetization $\left(4 \pi \mathrm{M}_{\mathrm{eff}}=4 \pi \mathrm{M}_{\mathrm{s}}-\mathrm{H}_{\perp}\right.$, where $\mathrm{H}_{\perp}$ is the perpendicular uniaxial anisotropy field) increases linearly with the annealing temperature leading to negative perpendicular anisotropy which enhances the in-plane easy axis. This effect is more pronounced for the $10 \mathrm{~nm}$ thick film: the slop of this linear dependence, in the range $415^{\circ} \mathrm{C}-615^{\circ} \mathrm{C}$, decreases from $32 \mathrm{Oe} /{ }^{\circ} \mathrm{C}$ to 9 $\mathrm{Oe} /{ }^{\circ} \mathrm{C}$ respectively for $10 \mathrm{~nm}$ and $50 \mathrm{~nm}$ thick CFA films. The origin of this effect is the interface $\mathrm{CFA} / \mathrm{MgO}$ that is improved with increasing the annealing temperature. Similar trend has been observed for CFA thin films deposited on $\mathrm{MgO}$ [5] where $4 \pi \mathrm{M}_{\text {eff }}$ increases with decreasing film thickness at fixed $\mathrm{T}_{\mathrm{a}}$. This thickness dependence anisotropy was also observed in the structures based on $\mathrm{CoFeB} / \mathrm{MgO}$ [7], but unlike $\mathrm{CFA} / \mathrm{MgO}$, the $\mathrm{CoFeB} / \mathrm{MgO}$ interface favours a perpendicular magnetization.

The typical angular dependence of resonance fields, shown in figure 5 for a thickness of $10 \mathrm{~nm}$ and $50 \mathrm{~nm}$ at 8 $\mathrm{GHz}$ driven frequency, reveals the presence of the uniaxial anisotropy in contrast to CFA grown on $\mathrm{MgO}$ substrates where a superposition of uniaxial and fourfold anisotropies has been observed [5]. This disappearance of the fourfold anisotropy for CFA grown on $\mathrm{Si}$ is directly correlated to the presence of in-plane polycrystalline structure due to the amorphous $\mathrm{SiO}_{2}$ layer.

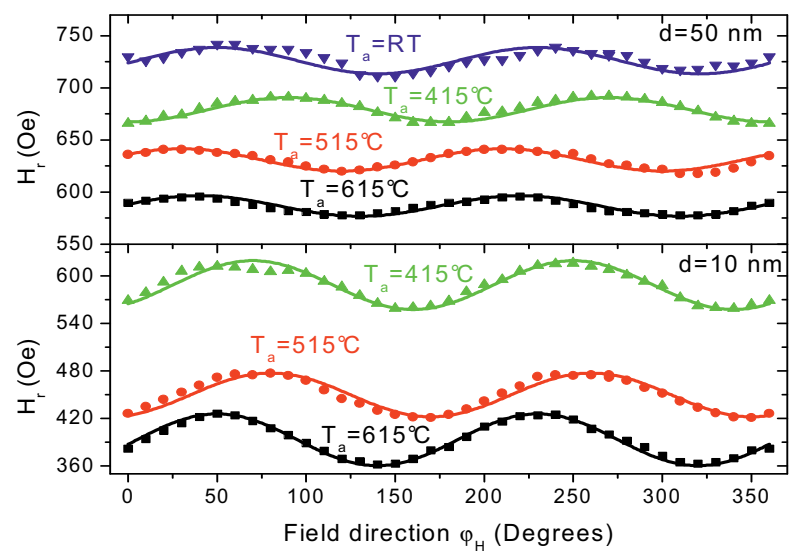

Fig. 5. (Color on line) Variations of the resonance fields $\left(H_{r}\right)$, as function of the angle between the applied field and the substrate edge $\left(\varphi_{\mathrm{H}}\right)$, of CFA thin films of thickness $\mathrm{d}=10 \mathrm{~nm}$ and $\mathrm{d}=50$ $\mathrm{nm}$ annealed at different temperatures. Solid lines indicate the fit using model described in [5].

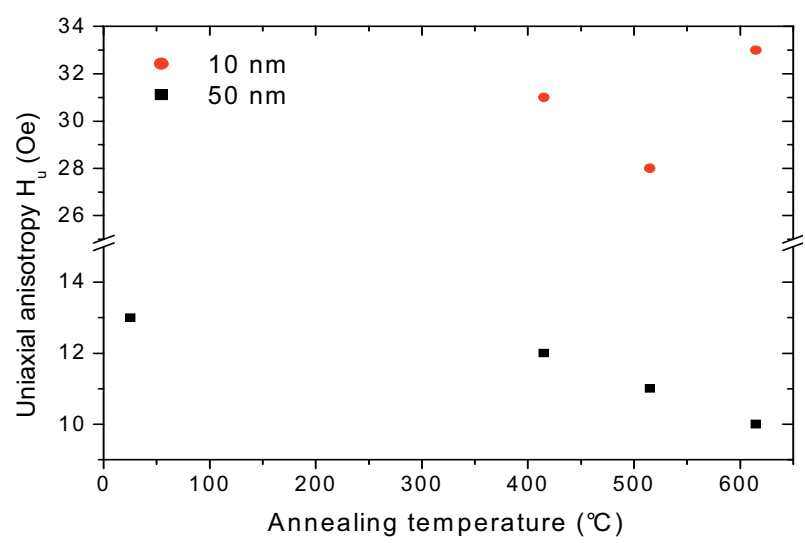

Fig. 6. (Color on line) Annealing temperature dependence of the uniaxial anisotropy field for $10 \mathrm{~nm}$ and $50 \mathrm{~nm}$ thick CFA films.

The anisotropy fields $\left(\mathrm{H}_{\mathrm{u}}=2 \mathrm{~K}_{\mathrm{u}} / \mathrm{M}_{\mathrm{s}}\right.$, where $\mathrm{K}_{\mathrm{u}}$ is the uniaxial anisotropy constant) extracted for the best fit of FMR data by the model described in [5] are shown on figure 6 . The $10 \mathrm{~nm}$ thick film presents a higher $\mathrm{H}_{\mathrm{u}}$ but no regular effect of annealing temperature on $\mathrm{H}_{\mathrm{u}}$ is observed, in contrast to $50 \mathrm{~nm}$ thick film where $\mathrm{H}_{\mathrm{u}}$ decreases with increasing $\mathrm{T}_{\mathrm{a}}$. As shown in figure 6 , the direction of the uniaxial anisotropy easy axis changes with thickness and with annealing temperature. The origin of this anisotropy is not clear but it is probably due to the strain induced by the lattice mismatch. 
The FMR linewidth is a measure of the relaxation rate of the magnetization and is related to the magnetic damping. This linewidth is caused by two mechanisms: the intrinsic damping of the magnetization and extrinsic contributions [8] (such as two magnons scattering, mosaicity,...). The angular and frequency dependences of the FMR linewidth provide information about these magnetic damping mechanisms. Therefore, the field peak to peak FMR linewidth, defined as the field difference between the extrema of the sweep-field measured FMR spectra, has been investigated as function of the annealing temperature.

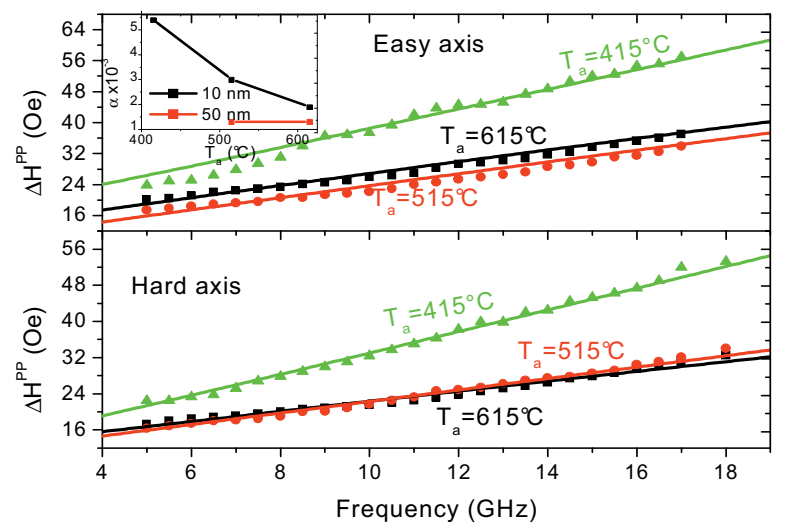

Fig. 7. (Color on line) Frequency dependence of the peak to peak filed FMR linewidth $\left(\Delta \mathrm{H}^{\mathrm{PP}}\right)$, using an applied filed along the easy and the hard axes, of the $10 \mathrm{~nm}$ thick CFA film annealed at different temperatures. Solid lines refer to the fit using the model described in [5]. The inset shows the annealing temperature dependence of the Gilbert damping parameter of the $10 \mathrm{~nm}$ and $50 \mathrm{~nm}$ CFA thin films.

Figure 7 presents the frequency dependence of FMR linewidth, for an applied field parallel to the easy and the hard axes, of $10 \mathrm{~nm}$ thick CFA annealed at various temperatures. It shows that the linewidth increases with decreasing $T_{a}$ maybe due to the enhancement of the chemical order with increasing $\mathrm{T}_{\mathrm{a}}$. The observed angular and frequency dependences of the field linewidth have been analyzed conjointly by considering intrinsic, two magnon scattering and inhomogeneities contributions given by the model presented in [5] and using the similar method described in [5]. The inset in figure 7 shows the $\mathrm{T}_{\mathrm{a}}$-dependence of the Gilbert damping constant $(\alpha)$. The $50 \mathrm{~nm}$ thick CFA film, annealed at $615^{\circ} \mathrm{C}$, shows a very low $\alpha$ of 0.0013 , which is comparable to that of $\mathrm{FeV}$ considered as the ferromagnetic metal having the lowest $\alpha$ [9]. However, the $10 \mathrm{~nm}$ thick sample shows relatively large values of $\alpha$ which increase with decreasing $T_{a}$. The reason for both these large values for thinner films and the increase of $\alpha$ with decreasing $\mathrm{T}_{\mathrm{a}}$, is most probably due to lower chemical order degree [10].

\section{Conclusions}

$\mathrm{Co}_{2} \mathrm{FeAl}$ films of $10 \mathrm{~nm}$ and $50 \mathrm{~nm}$ were prepared by sputtering on $\mathrm{Si}(001)$ substrates and annealed at various temperatures. They show both an out-of-plane and an inplane polycrystalline structure. The lattice parameter decreases with increasing annealing temperature due to the enhancement of the residual strain. VSM hysteresis loops obtained with different field orientations revealed that the magnetization at saturation increases slightly with the annealing temperature and the easy axis coercive field decreases with increasing annealing temperature. The microstrip ferromagnetic resonance (MS-FMR) has been used to study the dynamic properties. The MS-FMR has been adjusted by a model to determine the most relevant parameters. The in-plane uniaxial anisotropy field, present in all the samples, increases with decreasing annealing temperature. The effective magnetization shows drastically increase with the annealing temperature due to the enhancement of the $\mathrm{CFA} / \mathrm{MgO}$ interface quality. The angular and frequency dependences of the FMR linewidth, which increases with the decreasing annealing temperature, are governed by two magnon scattering and by a Gilbert damping which decreases with the increasing annealing temperature due to the chemical disorder.

\section{Acknowledgments}

This work has been partially supported by CNCSIS UEFISCSU, project number PNII - IDEI No.4/2010, code ID106 and by POS CCE ID. 574, code SMIS-CSNR 12467.

\section{References}

1. D. C. Ralph, M. D. Stiles, J. Magn. Magn. Mat. 320, 1190 (2008)

2. H. Jaffrès, A.Fert, J. Appl. Phys. 91, 8111 (2002)

3. G. Schmidt, J. Phys. D: Appl. Phys. 38, R107 (2005)

4. W. H. Wang, H. Sukegawa, and K. Inomata, Phys. Rev. B 82, 092402 (2010)

5. M. Belmeguenai,H. Tuzcuoglu, M. S. Gabor, T. Petrisor Jr. C. Tiusan, D. Berling,F. Zighem, T. Chauveau, S. M. Chérif, and P. Moch, Phys. Rev. B, 87, 184431 (2013)

6. M. S. Gabor, T. Petrisor Jr., and C. Tiusan, M. Hehn and T. Petrisor, Phys. Rev. B, 84, 134413 (2011)

7. S. Ikeda, K. Miura, H. Yamamoto, K. Mizunuma, H. D. Gan. M. Endo, S. Kanai, J. Hayakawa, F. Matsukura, and H. Ohno, Nature Mater. 9, 721 (2010)

8. Kh. Zakeri, J. Lindner, I. Barsukov, R. Meckenstock, M. Farle, U. von Hörsten, H. Wende, W. Keune, J. Rocker, S. S. Kalarickal, K. Lenz, W. Kuch, and K. Baberschke, Phys. Rev. B 76, 104416 (2007)

9. C. Scheck, L. Cheng, I. Barsukov, Z. Frait, and W. E. Bailey, Phys. Rev. Lett. 98, 117601 (2007).

10. S. Mizukami, D. Watanabe, M. Oogane, Y. Ando, Y. Miura, M. Shirai and T. Miyazaki, J. Appl. Phys.105, 07D306 (2009) 